\title{
22. Zur Karbildung im Hidaka-Gebirge, Hokkaido, Japan
}

\author{
Von Masao Minato und Seiji Hashimoto \\ (Comm. by H. YABE, M.J.A., Feb. 12, 1954)
}

Das Vorhandensein der Kartopographie im Hidaka-Gebirge, Hokkaido, ist den japanischen Geologen nichts Neues. Zuerst, schon vor Jahren, haben die Alpinisten des Hokkaido-Universität diese dort entdeckt. ${ }^{1)}$ Infolge des Berichtes dieser Alpinisten haben die Geologen ihre Untersuchungen begonnen; bedauerlicherweise, aber, bisher existieren da nur zwei oder drei kurze Berichte darüber. ${ }^{2}$ Nun unsere Untersuchungen an Ort und Stelle in der letzten Zeit haben die Verbreitungsorte, und die geologische und stratigraphische Nature der Kare klar erwiessen.

Das Hidaka-Gebirge erstreckt sich durch den südlichen Teil Hokkaidos und erreicht eine Höhe bis zu $2000 \mathrm{~m}$. Geologisch besteht es aus mannigfaltigen Tiefengesteinen, Migmatiten, sowie jurassischen und präjurassischen Formationen.

Gegenwärtig gibt es noch viele Berghöhe im Hokkaido, die höher als das Hidaka-Gebirge sind, wie, z.B., die Vulkane Daisetsu, Tokachi, Tomuraushi, usw., die zu Beginn des Alluvium erschienen und heute noch tätig sind. Im Diluvium, aber, war das Hidaka-Gebirge das höchste auf der ganzen Hokkaido; damals bildet das Zentralgebirge, wo jetzt verschiedene oben-genannte Vulkane liegen, ein sanft steigendes Plateau, beinahe $1000 \mathrm{~m}$ hoch. Auf den Kurilen sowie als auf der Halbinsel Kamtschatka, gab es auch noch keine hohen Vulkane in diluviale Zeit, und deshalb blieb das Hidaka-Gebirge am höchsten im ganzen nördlichen Japan und im Kurilen Bogen.

Die fraglichen Kare befinden sich meist an der östlichen Seite des Hauptgebirgeszuges, aber auch die Nebenzuge weissen an ihre östlichen Seite solche Kare auf. Die Höhenlage des Karbodens ist durchschnittlich $1640 \mathrm{~m}$, obwohl in seltenen Fällen ist er örtlich mehr oder weniger davon verschieden. Der Karboden ist besonders weit im Gebiet des Poroshiri-dake im nördlichen Hidaka-Gebirge. Die Karwand ist sehr steil; aber in vielen Fällen ist sie verdeckt von einer dicken Schicht von Karschutt.

Der Rand des Karbodens ist etwas erhöht und fällt dann in die

1) K. Yamaguchi: Zur Entdeckung der karförmigen Topographie im HidakaGebirge, Hokkaido. Berg und Ski (auf japanisch), Nr. 88 (1928).

2) Y. Sasa: Geomorphology of the Japanese High Mountains. Third Report. Glacial Topography in the Hidaka Mountain Range, Hokkaido. Proc. Imp. Acad., 10, 218 (1934). 


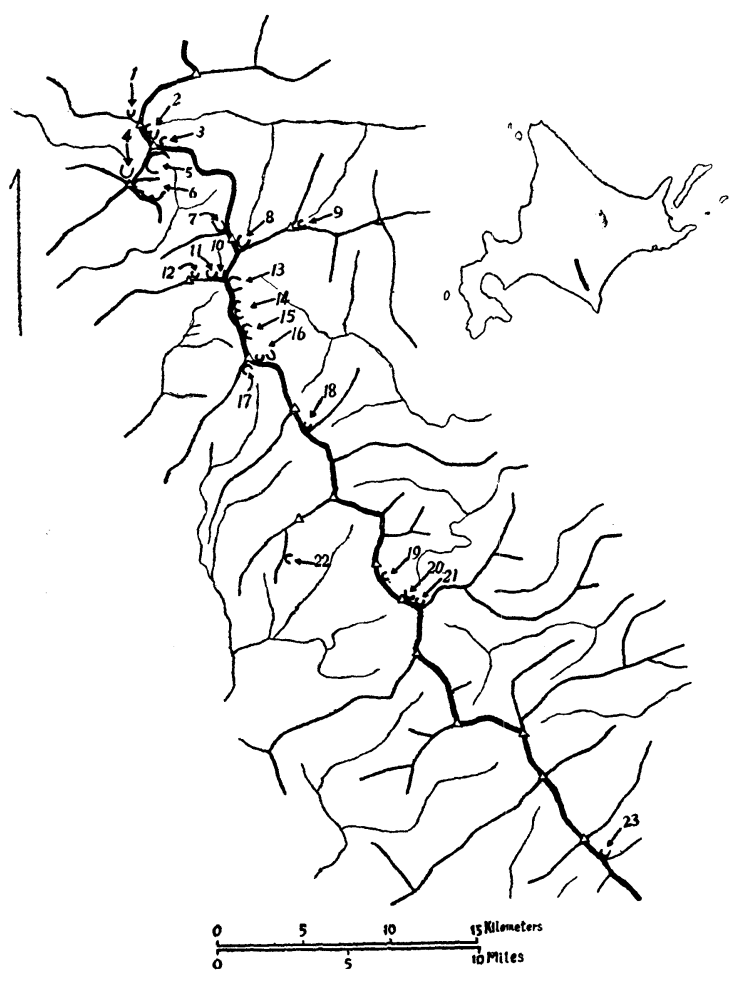

1 Kita(Nord)-Tottabetsu-dake

2 Tottabetsu-dake, A

$3 \quad, \quad$, B

4 Kita(Nord)-Poroshiri-dake

5 Numa (der See), A

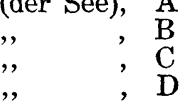

6 Higashi'(Ost)-PoroshiriA
B
D
daki-
dake ," dake, $\mathrm{A}$

7 Kita(Nord)-Esaomantot-

8 Higashi(Ost)-Esaomantottabetsu-dake

9 Satsunai-dake

10 Namewakka-dake, A

13 Bunkiten (Scheideweg)

14 Spitz $1900 \mathrm{~m}$, A

15 , B

16 Kamiekuchikaushi-yama, $17 \begin{array}{r}\text { Nunosawa, A } \\ \text { Kamiekuchikaushi-yama, B } \\ \text { Hachinosawa, A }\end{array}$

18 Spitz 1823 '

Hachinosawa, A

19 Petegari-dake, C

20 , , B

21 Shibichari-yama

23 Toyaoi-dake

fraglich

Abb. 1. Die Verbreitung des Kar im Hidaka-Gebirge

(Dake und Yama deutet auf japanisch der Berg)

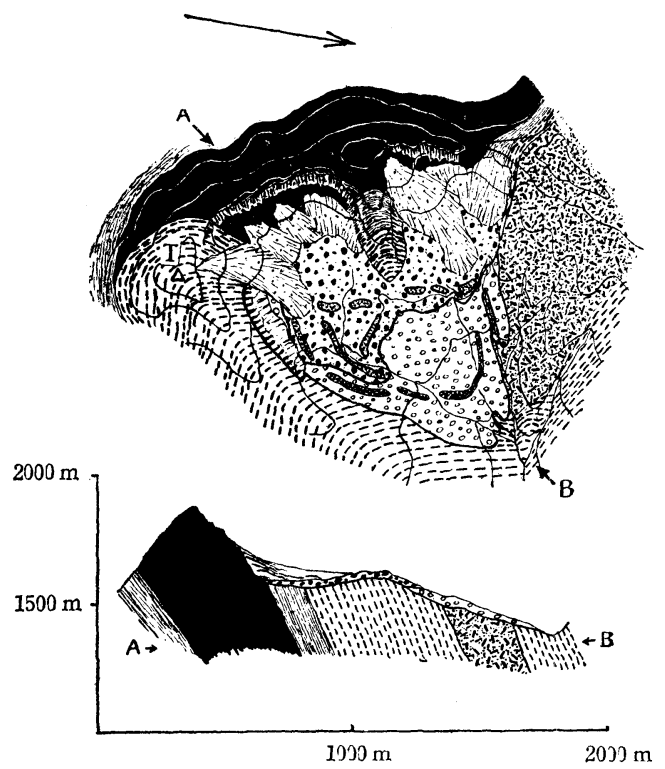

\begin{tabular}{|c|c|}
\hline 10 & Karschutt (Alluvium-Schutt) \\
\hline 8 & Moränen! Amphitheater \\
\hline & Neue Moräne \\
\hline & Alte Moräne \\
\hline & Dunit \\
\hline 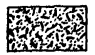 & Gabbro \\
\hline & Amphibolit \\
\hline 漬 & Biotitgneis \\
\hline
\end{tabular}

Abb. 2. Schematische Darstellung der Topographie und Geologie, in der Nähe des Kar A und B, bei Tottabetsu-dake 
Moräne ab. Die Moräne sind fast ohne Ausnahme zweistufig und liegen in seiner Höhe zwischen 1600 und $1400 \mathrm{~m}$.

Nicht nur topographisch, sondern auch stratigraphisch sind sie zweistufig und die niederen Moräne sind älter als die höheren.

Die Bestandteile der Moräne sind örtlich verschieden; z.B., im Falle des Tottabetsu-dake-Kar besteht die niedere Moräne ausschliesslich aus Biotitgneis, während die höhere Moräne aus Dunit besteht. Dabei liegt Dunit mehr inseitig als Biotitgneis, wie der NO-SW gerichtete geologische Durchschnitt (Abb. 2) zeigt.

Überdies ist es sehr beachtenswert, dass nicht nur die Moräne zweistufig, sondern auch die Karwand zuweilen zweiteilig ist, dabei schabt die neue Wand die ältere ab.

Die Schlussfolgerung ist, dass eine zweimalige Karbildung dort erfolgt sein muss. Die erste Verkarung schabte den Gneis ab, und die zweite schnitt durch bis zum Dunit ein.

Ein Vergleich der Hidaka-Karbildung mit jener Europas ist nicht leicht; aber auf den mannigfaltigen Grunden im Diluvium Japans, scheint es uns sehr wahrscheinlich dass die älte Karbildungszeit mit dem Riss und die neue vielleicht mit dem Würm entspricht, dafür möchten wir in der nähe Zukunft äussern.

Zum Schluss fühlen wir die Pflicht, unseren verehrten Lehrer Herrn Em. Prof. H. Yabe für seine gütige Anleitung zu danken. 